\title{
Immunohistochemical detection of GLUT-1 can discriminate between reactive mesothelium and malignant mesothelioma
}

\author{
Yasufumi Kato ${ }^{1,2}$, Koji Tsuta ${ }^{1}$, Kunihiko Seki ${ }^{1}$, Akiko Miyagi Maeshima ${ }^{3}$, \\ Shunichi Watanabe ${ }^{2}$, Kenji Suzuki ${ }^{2}$, Hisao Asamura ${ }^{2}$, Ryosuke Tsuchiya ${ }^{2}$ \\ and Yoshihiro Matsuno ${ }^{1}$ \\ ${ }^{1}$ Clinical Laboratory, National Cancer Center Hospital, Tokyo, Japan; ${ }^{2}$ Thoracic Surgery Divisions, National \\ Cancer Center Hospital, Tokyo, Japan and ${ }^{3}$ Pathology Division, National Cancer Center Research Institute, \\ Tokyo, Japan
}

\begin{abstract}
The separation of benign reactive mesothelium (RM) from malignant mesothelial proliferation can be a major challenge. A number of markers have been proposed, including epithelial membrane antigen, p53 protein, and P-glycoprotein. To date, however, no immunohistochemical marker that allows unequivocal discrimination of RM from malignant pleural mesothelioma (MPM) has been available. A family of glucose transporter isoforms (GLUT), of which GLUT-1 is a member, facilitate the entry of glucose into cells. GLUT-1 is largely undetectable by immunohistochemistry in normal epithelial tissues and benign tumors, but is expressed in a variety of malignancies. Thus, the expression of GLUT-1 appears to be a potential marker of malignant transformation. Recently, in fact, some studies have shown that GLUT-1 expression is useful for distinguishing benign from malignant lesions. The purpose of the present study was to evaluate the diagnostic utility of GLUT-1 expression for diagnostic differentiation between RM and MPM. Immunohistochemical staining for GLUT-1 was performed in $\mathbf{4 0}$ cases of RM, $\mathbf{4 8}$ cases of MPM, and $\mathbf{5 8}$ cases of lung carcinoma. Immunohistochemical GLUT-1 expression was seen in 40 of $40(100 \%)$ MPMs, and in all cases the expression was demonstrated by linear plasma membrane staining, sometimes with cytoplasmic staining in addition. GLUT-1 expression was also observed in 56 out of $58(96.5 \%)$ lung carcinomas. On the other hand, no RM cases were positive for GLUT-1. GLUT-1 is a sensitive and specific immunohistochemical marker enabling differential diagnosis of RM from MPM, whereas it cannot discriminate MPM from lung carcinoma.

Modern Pathology (2007) 20, 215-220. doi:10.1038/modpathol.3800732; published online 22 December 2006
\end{abstract}

Keywords: Glut-1; reactive methothelium; malignant pleural mesothelioma; immunohistochemistry; lung carcinoma

The separation of benign reactive mesothelium (RM) from malignant mesothelial proliferation can be a major challenge. The common cytomorphological features associated with malignancy, such as high cellularity/proliferation, marked cytonuclear atypia and high mitotic rate are of very limited use in this setting. Thus, it is sometimes very difficult, or almost impossible even for expert pathologists to make a definite diagnosis of malignant mesothelioma, especially in small specimens, unless there is unequivocal invasion of adjacent tissues by tumor cells. ${ }^{1}$ On the other hand, early diagnosis of

Correspondence: Dr Y Matsuno, MD, Clinical Laboratory Division, National Cancer Center Hospital, 1-1, Tsukiji 5-chome, Chuo-ku, Tokyo 104-0045, Japan.

E-mail: ymatsuno@ncc.go.jp

Received 30 August 2006; accepted 23 October 2006; published online 22 December 2006 malignant pleural mesothelioma (MPM) in small closed pleural biopsy samples, or by cytology, is crucial for patient management and may facilitate the avoidance of invasive surgical procedures.

A number of immunohistochemical markers have been proposed to assist conventional morphological diagnosis, including epithelial membrane antigen (EMA $^{2-5}$ p53 protein, ${ }^{2-11}$ and P-glycoprotein., ${ }^{2,5,12}$ Other markers tested have included Bcl-2, ${ }^{2,3,13}$ platelet-derived growth factor receptor (PDGF-R) $\beta$ chain $^{2,5,8}$ and desmin. ${ }^{2}$ To date, however, no single immunohistochemical marker that can unequivocally discriminate RM from MPM has been available.

GLUT-1 is one of 14 members of the mammalian facilitative glucose transporter (GLUT) family of passive carriers that function as an energy-independent system for transport of glucose down a concentration gradient. ${ }^{14}$ GLUT-1 is not detectable 
in a large proportion of cells from normal tissues and benign lesions, except for erythrocytes, germinal cells of the testis, renal tubules, perineurium of peripheral nerves, endothelial cells in blood-brain barrier vessels, and placenta (trophoblasts and capillaries). ${ }^{15,16}$ In contrast, GLUT-1 is expressed in a variety of carcinomas such as those of the breast, head and neck, bladder, renal cells, and lung. ${ }^{15-24}$ Previous reports suggest that the expression of GLUT-1 may be a potential marker for malignancy.

Recently, some studies have shown that GLUT-1 expression is useful for resolving the common diagnostic dilemma of distinguishing benign from malignant lesions. ${ }^{25,26}$ Although a few studies have demonstrated that GLUT-1 is useful for distinguishing RM from metastatic adenocarcinoma in body cavity effusions, ${ }^{27-29}$ the study cohorts did not include MPM. Using immunohistochemistry, Godoy et $a 1^{16}$ analyzed coexpression of GLUT-1 and other GLUT isoforms (GLUT-2 to -6 and GLUT-9) in a variety of benign and malignant tumors, and demonstrated that two of four MPMs were positive for GLUT-1. However, they did not analyze reactive and normal mesothelium.

The purpose of the present study was to evaluate the diagnostic utility of GLUT-1 detection for differential diagnosis between RM and MPM.

\section{Materials and methods}

\section{Case Selection}

The materials for the present study were extracted from cases deposited in the pathology files of the National Cancer Center Hospital, Tokyo, between 1971 and 2005. They comprised 40 cases of RM, 48 cases of MPM (epithelioid, 36 cases; biphasic, 11 cases; sarcomatoid, 1 case), and 58 cases of lung carcinoma (squamous cell carcinoma, 28 cases; adenocarcinoma, 30 cases). All diagnoses had been made on the basis of conventional histopathologic features evident in slide preparations stained with hematoxylin and eosin, some special stains, and immunohistochemical techniques available at that time. ${ }^{30,31}$ In the present study, immunohistochemistry for D2-40 and calretinin was added for all cases to confirm the identity of mesothelial cells (see below).

\section{Immunohistochemistry}

For immunohistochemical staining, 5 - $\mu$ m-thick sections were deparaffinized and treated with $3 \%$ hydrogen peroxide for 30 min to block endogenous peroxidase activity, followed by washing in deionized water for 2-3 min. Heat-induced epitope retrieval with Target Retrieval Solution (DAKO, Carpinteria, CA, USA) was performed for GLUT-1 and calretinin. After the slides had been allowed to cool at room temperature for $40 \mathrm{~min}$, they were rinsed with deionized water and then washed in phosphate-buffered saline for $5 \mathrm{~min}$. The slides were then stained by overnight incubation with primary antibodies against GLUT-1 (1:200, polyclonal, Dako), D2-40 (1:200, clone D2-40, Signet Laboratories, Dedham, MA, USA), and calretinin (1:100, polyclonal, Zymed, San Francisco, CA, USA). Immunoreactions were detected by the labeled streptavidin-biotin method, and visualized with 3 , 3 -diaminobenzidine, followed by counterstaining with hematoxylin. Appropriate positive and negative controls (red blood cells for GLUT-1) were used for each antibody. The area of GLUT-1 staining was evaluated on a sliding scale of 0 to $3+$ to represent the percentage of positive cells among mesothelial cells (indicated by D2-40 and calretinin immunostain) or tumor cells $(0=<1 \%, 1+=1-25 \%, 2+$ $=26-50 \%, 3+=>51 \%$ ). Immunohistochemical staining was scored independently by two observers (YK and KT).

\section{Results}

The results of immunohistochemistry are summarized in Table 1. GLUT-1 expression was demonstrated by distinct linear plasma membrane staining, sometimes with cytoplasmic staining in addition

Table 1 Immunoreactivity of GLUT-1

GLUT-1 positive (\%)

\begin{tabular}{lrc} 
& & \\
\hline Mesothelioma, all subtypes & 48 & $48(100)$ \\
$\quad$ Epithelioid & 36 & $36(100)$ \\
Biphasic & 11 & $10(90.9)^{\mathrm{a}} 7(63.6)^{\mathrm{b}}$ \\
Sarcomatoid & 1 & $1(100)$ \\
Reactive mesothelium & 40 & $0(0)$ \\
Lung carcinoma & 58 & $56(96.5)$ \\
Squamous cell carcinoma & 28 & $28(100)$ \\
Adenocarcinoma & 30 & $28(93.3)$
\end{tabular}

${ }^{\mathrm{a}}$ Epithelioid areas.

${ }^{\mathrm{b}}$ Sarcomatoid areas.
Staining area

\begin{tabular}{cccc}
\hline 0 & $1+$ & $2+$ & $3+$ \\
\hline 0 & 15 & 15 & 18 \\
0 & 9 & 12 & 15 \\
$1^{\mathrm{a}} 4^{\mathrm{b}}$ & $6^{\mathrm{a}} 3^{\mathrm{b}}$ & $3^{\mathrm{a}} 2^{\mathrm{b}}$ & $1^{\mathrm{a}} 2^{\mathrm{b}}$ \\
0 & 1 & 0 & 0 \\
40 & 0 & 0 & 0 \\
2 & 12 & 9 & 35 \\
0 & 1 & 3 & 24 \\
2 & 11 & 6 & 11 \\
\hline
\end{tabular}




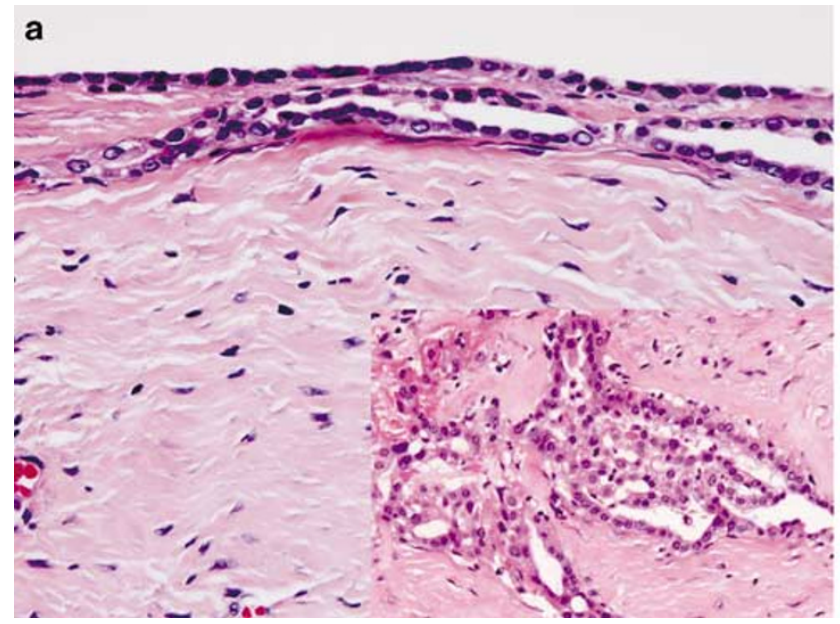

b

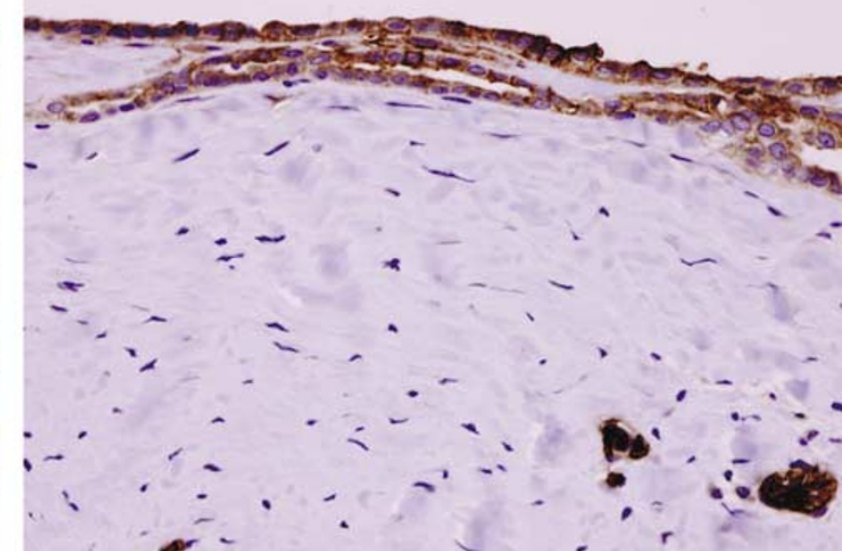

Figure 1 (a) In the surface area, the tumor cells showed bland cytologic atypia, nevertheless malignant mesothelioma(HE stain, $\times 10)$. Inset: the tumor cells arranged complex branching tubular formation (HE stain, $\times 10$ ). (b) Most of the tumor cells in the epithelioid MPM were positive for GLUT-1 and red blood cells were served as internal positive control $(\times 10)$.
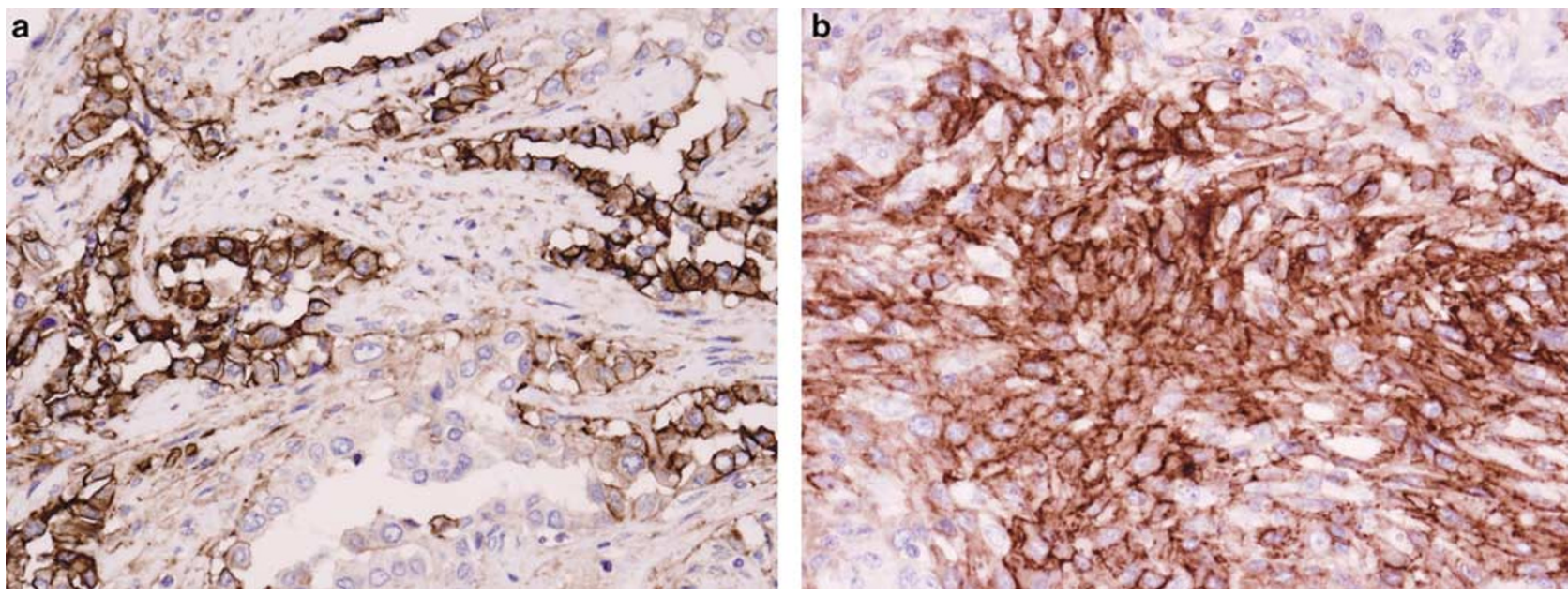

Figure 2 (a) More than half of the epithelioid tumor cells were positive for GLUT-1 $(\times 10)$. (b) Most of the sarcomatoid tumor cells were positive for GLUT-1 $(\times 10)$. The immunoreactivity was observed as distinct linear plasma membrane staining, with weak cytoplasmic staining in addition.

Table 2 GLUT-1 immunoreactivity acording to MPM histological subtype

\begin{tabular}{|c|c|c|c|c|c|c|}
\hline & \multirow[t]{2}{*}{$\mathrm{n}$} & \multirow[t]{2}{*}{ GLUT-1-positive (\%) } & \multicolumn{4}{|c|}{ Staining area } \\
\hline & & & 0 & $1+$ & $2+$ & $3+$ \\
\hline Epithelioid area & 47 & $46(97.8)$ & 1 & 15 & 15 & 16 \\
\hline Sarcomatoid area & 12 & $8(66.7)$ & 4 & 4 & 2 & 2 \\
\hline
\end{tabular}

(Figure 1a and b). GLUT-1 immunoreactivity was seen in 48 of $48(100 \%)$ MPM cases, whereas no RM cases were positive for GLUT-1.

We also evaluated GLUT-1 immunoreactivity according to histological subtype, as shown in Table 2. Immunoreactivity was observed in 46 of
$47(96.7 \%)$ epithelioid mesothelioma (Figure 2a) including epithelioid areas of biphasic mesothelioma, and in seven of $12(66.7 \%)$ sarcomatoid mesothelioma (Figure 2b) including sarcomatoid areas of biphasic mesothelioma. However, immunoreactive cells more than half of tumor cell was only 16 of $47(34 \%)$ of epithelioid mesothelioma including epithelioid areas of biphasic mesothelioma, and two of $12(14.1 \%)$ of sarcomatoid mesothelioma including sarcomatoid areas of biphasic mesothelioma. The GLUT-1-positive cells varied from a few cells to almost all cells in the clusters, but no characteristic staining pattern was observed in MPM.

GLUT-1 immunoreactivity was also seen in 56 of $58(96.5 \%)$ cases of lung carcinoma. According to histological subtype, immunoreactivity was 

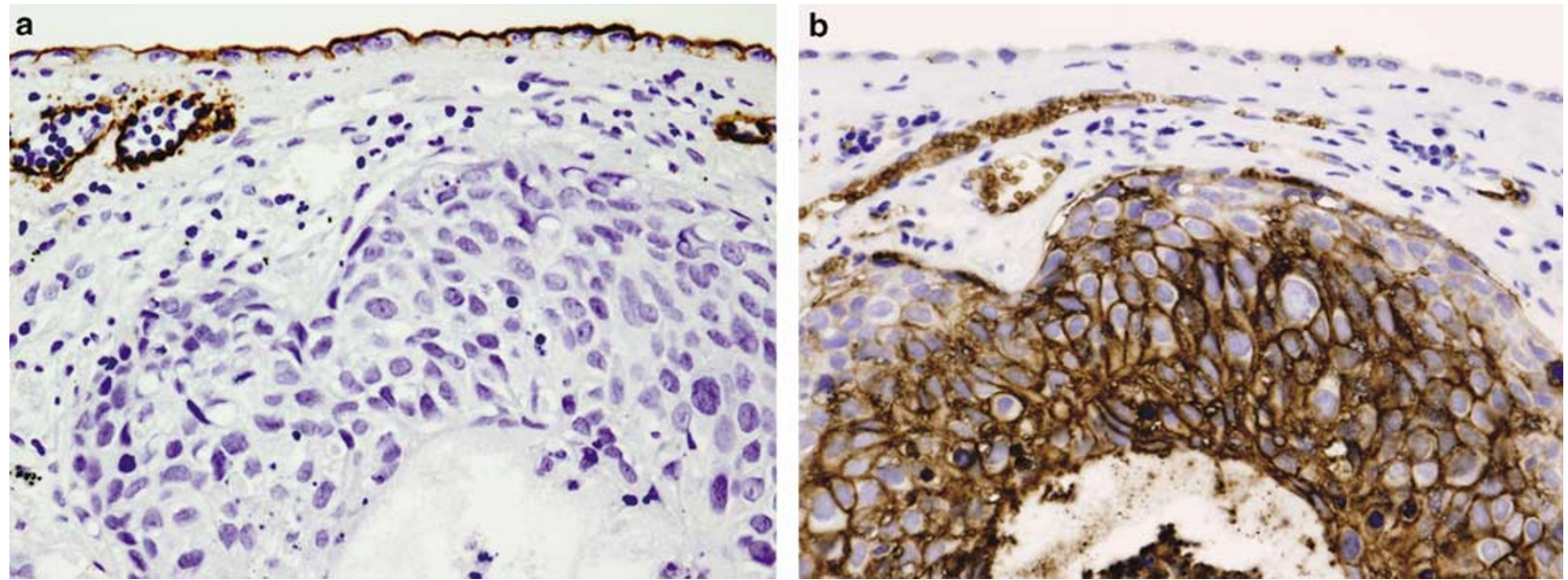

Figure 3 (a) D2-40 immunoreactivity was observed in the RM and lymph vessels beneath the pleura, but no immunoreactivity was observed in the poorly differentiated squamous cell carcinoma $(\times 10)$. (b) Most of the tumor cells without peripheral lesion in of the poorly differentiated squamous cell carcinoma were positive for GLUT-1 (red blood cells were served as internal positive control). On the other hand, RM showed no immunoreactivity for GLUT-1 ( × 10).

observed in 28 of $28(100 \%)$ cases of squamous cell carcinoma (Figure 3a and b) and 28 of 30 (93.3\%) cases of adenocarcinoma. In squamous cell carcinoma, the area of positive staining was $3+$ in 24 of 28 $(85.7 \%)$ cases, compared with only 11 of $30(36.7 \%)$ in cases of adenocarcinoma. Also in squamous cell carcinoma, a characteristic staining pattern was observed; tumor cells showed more intensely positive staining in the central area of tumor nests than in the peripheral area (Figure $3 b$ ).

\section{Discussion}

Morphologic differentiation between RM and MPM in small specimens can be a diagnostic challenge. The difficulty is compounded when neoplastic cells demonstrate only slight atypia. In addition, there are currently no reliable markers that allow immunohistochemical discrimination between RM and MPM. In the present study, we clearly demonstrated that GLUT-1 is a sensitive and specific immunohistochemical marker that can differentiate RM from MPM. To our knowledge, this is the first report to describe the usefulness of GLUT-1 immunostaining for discriminating between RM and MPM.

Elevated levels of expression or activation of GLUT-1, or both, have been shown to be associated with transformation of cells and malignancy, and to be modified by changes in the physiological microenvironment in tissues. ${ }^{32,33}$ High GLUT-1 expression correlates with increased metabolism and glucose utilization in a number of normal tissues, and this transporter is overexpressed in a variety of human tumors. ${ }^{15,16}$ Increased expression of GLUT-1 is also seen in conditions that induce greater dependency on glycolysis as an energy source, such as ischemia, hypoxia, or both. ${ }^{34}$ These data suggest that overexpression of GLUT-1 may play an important role in the survival of tumor cells by maintaining an adequate energy supply to support their high metabolism and rapid growth in an often less-thanideal physiological environment. ${ }^{35}$

GLUT-1 expression has been revealed in a variety of carcinomas, such as those of the breast, head and neck, bladder, and renal cells. ${ }^{15-19,23}$ In the lung, about $34.3-100 \%$ of lung adenocarcinomas ${ }^{16,20-22,24}$ and $100 \%$ of lung squamous cell carcinomas ${ }^{20-22,24}$ are reported to express GLUT-1 at the primary site. With regard to MPM, only one article has describe that two of four studied cases were positive for GLUT- $1 .{ }^{16}$ In the present study, GLUT-1 immunoreactivity was observed in all MPMs and 56 out of 58 $(96.5 \%)$ cases of lung carcinoma. These results indicate that GLUT-1 cannot discriminate between MPM and lung carcinoma. Therefore, additional appropriate positive and negative mesothelial markers are needed in order to differentiate between MPM and lung carcinoma. ${ }^{31}$

The heterogeneity of GLUT-1-positive areas has been reported previously. In squamous cell carcinoma, cells in the center of cancer nests, close to the necrotic area, were stained more strongly than those in peripheral areas. In adenocarcinoma, poorly differentiated areas such as the solid central area were stained more strongly than well differentiated areas such as those showing lepidic growth. ${ }^{20-22,24}$ In the present study, more than half of all tumor cells were positive for GLUT-1 in $37.5 \%$ of MPMs, $85.7 \%$ of lung squamous cell carcinomas, and $36.7 \%$ of lung adenocarcinomas. These results indicate that GLUT-1 negativity in small samples such as those obtained by biopsy does not exclude malignancy, and that positive immunoreactivity for GLUT-1 may be an aid to accurate diagnosis of malignancy.

The GLUT-1 positivity rate in RM has been reported to be $0 \%$ (present study and Afify et $a l^{29}$ ), $3 \%$ (Zimmerman et $a 1^{28}$ ), and 20\% (Burstein et $a l^{27}$ ). 
However, Zimmerman et al and Burstein et al reported that GLUT-1-positive cells of RM showed equivocal-to-weak staining and were easily distinguishable from unequivocal positivity of other cell types, so that the specificity of GLUT-1 was not diminished. According to them, a number of 'falsepositive' cases occurred in patients with cirrhosis. The RM resulting from cirrhosis may be prompted by glucose intake to compensate for the unfavorable environment in effusion. Our cohort of RM consisted of surgically resectable cases within the physiological range or without effusion.

Positron emission tomography (PET) measurements of fluorodeoxyglucose (FDG) accumulation in different animal tumors has shown a correlation between tracer FDG uptake and the GLUT-1 mRNA content. GLUT-1 has been found to be overexpressed in tumor cells and to promote glucose metabolism and FDG accumulation in humans. ${ }^{22,24}$ In MPM, Carretta et $a l^{36}$ have reported that FDG-PET can differentiate RM from MPM. These findings are consistent with the present immunohistochemical results.

In summary, GLUT-1 appears to be a sensitive and specific marker for differentiating between RM and MPM, although it is unable to discriminate between MPM and lung carcinoma.

\section{Acknowledgement}

This work is supported in part by Special Coordination Funds for Promoting Science and Technology of Japan.

\section{References}

1 Allen TC, Cagle PT, Churg AM, et al. The separation of benign and malignant mesothelial proliferations. Am J Surg Pathol 2000;24:1183-2000.

2 Attanoos RL, Gibbs AR. Pathology of malignant mesothelioma. Histopathology 1997;30:403-418.

3 Cury PM, Butcher DN, Corrin B, et al. The use of histological and immunohistochemical markers to distinguish pleural malignant mesothelioma and in situ mesothelioma from reactive mesothelial hyperplasia and reactive pleural fibrosis. J Pathol 1999;189: 251-257.

4 Salas A, Fernandez-Banares F, Casalots J, et al. Utility of epithelial membrane antigen and p53 in the differential diagnosis of benign reactive processes from malignancy in pleural biopsy specimens. Virchows Arch 1999;435:286.

5 Roberts F, Harper CM, Downie I, et al. Immunohistochemical analysis still has a limited role in the diagnosis of malignant mesothelioma. Am J Clin Pathol 2001;116:253-262.

6 Kafiri G, Thomas DM, Shepherd NA, et al. p53 expression is common in malignant mesothelioma. Histopathology 1992;21:331-334.

7 Mayall FG, Goddard H, Gibbs AR. p53 immunostaining in the distinction between benign and malignant mesothelial proliferations using formalin-fixed paraffin sections. J Pathol 1992;168:377-381.

8 Ramael M, Buysse C, van den Bossche J, et al. Immunoreactivity for the $\mathrm{b}$ chain of the plateletderived growth factor receptor in malignant mesothelioma and non-neoplastic mesothelium. J Pathol 1992; 167:1-4.

9 Ramael M, Lemmens G, Eerdekens C, et al. Immunoreactivity for p53 protein in malignant mesothelioma and non-neoplastic mesothelium. J Pathol 1992;168: 371-375.

10 Cagle PT, Brown RW, Lebovitz RM. p53 immunostaining in the differentiation of reactive processes from malignancy in pleural biopsy specimens. Hum Pathol 1994;25:443-448.

11 Esposito V, Baldi A, De Luca A, et al. p53 immunostaining in differential diagnosis of pleural mesothelial proliferations. Anticancer Res 1997;17:733-736.

12 Ramael M, van den Bossche J, Buysse C, et al. Immunoreactivity for P-170 glycoprotein in malignant and in non-neoplastic mesothelium of the pleura arising the murine monoclonal antibody JSB-1. J Pathol 1992;167:5-8.

13 Segers K, Kumar-Singh S, Weyler J, et al. Immunoreactivity for bcl-2 protein in malignant mesothelioma and non-neoplastic mesothelium. Virchows Arch 1994;242:631-634.

14 Olson AL, Pessin JE. Structure, function, and regulation of the mammalian facilitative glucose transporter gene family. Annu Rev Nutr 1996;16:235-256.

15 Younes M, Lechago LV, Somoano JR, et al. Wide expression of the human erythrocyte glucose transporter Glut1 in human cancers. Cancer Res 1996;56: 1164-1167.

16 Godoy A, Ulloa V, Rodriguez F, et al. Differential subcellular distribution of glucose transporters GLUT1-6 and GLUT9 in human cancer: ultrastructural localization of GLUT1 and GLUT5 in breast tumor tissues. J Cell Physiol 2006;207:614-627.

17 Brown RS, Wahl RL. Overexpression of Glut-1 glucose transporter in human breast cancer. An immunohistochemical study. Cancer 1993;72:2979-2985.

18 Mellanen P, Minn H, Grenman R, et al. Expression of glucose transporters in head-and-neck tumors. Int J Cancer 1994;56:622-629.

19 Nagase Y, Takata K, Moriyama N, et al. Immunohistochemical localization of glucose transporters in human renal cell carcinoma. J Urol 1995;153:798-801.

20 Younes M, Brown RW, Stephenson M, et al. Overexpression of Glut1 and Glut3 in stage I nonsmall cell lung carcinoma is associated with poor survival. Cancer 1997;80:1046-1051.

21 Ito $\mathrm{T}$, Noguchi $\mathrm{Y}$, Satoh S, et al. Expression of facilitative glucose transporter isoforms in lung carcinomas: its relation to histologic type, differentiation grade, and tumor stage. Mod Pathol 1998;11:437-443.

22 Brown RS, Leung JY, Kison PV, et al. Glucose transporters and FDG uptake in untreated primary human non-small cell lung cancer. J Nucl Med 1999;40:556-565.

23 Chang S, Lee S, Lee C, et al. Expression of the human erythrocyte glucose transporter in transitional cell carcinoma of the bladder. Urology 2000;55:448-452.

24 Mamede M, Higashi T, Kitaichi M, et al. [18F]FDG uptake and PCNA, Glut-1, and Hexokinase-II expressions in cancers and inflammatory lesions of the lung. Neoplasia 2005;7:369-379. 
25 Weiner MF, Miranda RN, Bardales RH, et al. Diagnostic value of GLUT-1 immunoreactivity to distinguish benign from malignant cystic squamous lesions of the head and neck in fine-needle aspiration biopsy material. Diagn Cytopathol 2004;31:294-299.

26 Chandan VS, Faquin WC, Wilbur DC, et al. The utility of GLUT-1 immunolocalization in cell blocks: an adjunct to the fine needle aspiration diagnosis of cystic squamous lesions of the head and neck. Cancer 2006;108:124-128.

27 Burstein DE, Reder I, Weiser K, et al. GLUT1 glucose transporter: a highly sensitive marker of malignancy in body cavity effusions. Mod Pathol 1998;11:392-396.

28 Zimmerman RL, Goonewardene S, Fogt F. Glucose transporter Glut-1 is of limited value for detecting breast carcinoma in serous effusions. Mod Pathol 2001;14:748-751.

29 Afify A, Zhou H, Howell L, et al. Diagnostic utility of Glut-1 expression in the cytologic evaluation of serous fluids. Acta Cytol 2005;49:621-626.

30 Churg A, Roggli V, Galateau-Salle F, et al. Mesothelioma. In: Travis WD, Brambilla E, Muller-Hermelink HK,
Harris CC (eds). Pathology and Genetics: Tumors of the Lung, Pleura, Thymus and Heart. IARC: Lyon, France, 2004, pp 128-136.

31 Ordonez NG. Immunohistochemical diagnosis of epithelioid mesothelioma: an update. Arch Pathol Lab Med 2005;129:1407-1414.

32 Merrall NW, Plevin R, Gould GW. Growth factors, mitogens, oncogenes and the regulation of glucose transport. Cell Signal 1993;5:667-675.

33 Mueckler M. Facilitative glucose transporters. Eur J Biochem 1994;219:713-725.

34 Clavo AC, Brown RS, Wahl RL. Fluorodeoxyglucose uptake in human cancer cell lines is increased by hypoxia. J Nucl Med 1995;36:1625-1632.

35 Newsholme EA, Board M. Application of metabolic-control logic to fuel utilization and its significance in tumor cells. Adv Enzyme Regul 1991;31: 225-246.

36 Carretta A, Landoni C, Melloni G, et al. 18-FDG positron emission tomography in the evaluation of malignant pleural diseases-a pilot study. Eur J Cardiothorac Surg 2000;17:377-383. 